\title{
Prevalencia de resistencia a los anti-retrovirales en niños y adolescentes uruguayos infectados con el virus de la inmunodeficiencia humana en el Centro de Referencia VIH-SIDA. Centro Hospitalario Pereira Rossell. Montevideo-Uruguay
}

\author{
Virginia González’ y Stella Gutiérrez
}

Prevalence of antiretroviral resistance in Uruguayan children and adolescents infected with the human immunodeficiency virus in the Reference Center HIV-AIDS. Centro Hospitalario Pereira Rossell, Montevideo, Uruguay

Background: Primary and secondary antiretroviral (ARV) resistance rates of 15 and $40 \%$ respectively have been reported in worldwide. Its prevalence in Uruguay is unknown. Aim: To know the prevalence of ARV resistance in Uruguayan children under 15 years old infected with HIV that are controlled in the Centro Hospitalario Pereira Rossell between 2008 and 2016. Specific objectives: Quantify primary and secondary mutations, to identify variables associated with resistance; to describe if the result of the resistance test contributed to achieve undetectable viral load (VL). Methodos: Observational descriptive, longitudinal follow-up. Only children under 15 years with resistance test done between January first 2008 and December $31^{\text {th }} 2016$ were included in the study. Maternal and child variables. Results: Fifty six children were included. 36 children (64\%) had resistance tests prior to the initiation of ART and the other 20 children (36\%) due to therapeutic failure. Total resistance: $28.6 \%$ ( 16 children); $4(11.1 \%)$ children with primary mutations and $12(60 \%)$ secondary mutations. The test result changed the ARV plan in $15(26.7 \%)$ of the 56 children. The change achieved undetectable CV in 8 children at month 6 . The ART change was not associated with AIDS or death. Discussion: Prevalence studies are useful in making decisions about initial ARV treatment. The prevalence of primary mutations was similar to that published, while secondary prevalence was higher.

Keywords: HIV in pediatrics; genotypic resistance test; antiretroviral resistance.

Palabras clave: VIH en pediatría; test de resistencia genotípico; resistencia anti-retroviral.

\section{Introducción}

$\mathrm{E}$ 1 tratamiento de la infección por el virus de la inmunodeficiencia humana (VIH) ha tenido una gran evolución desde el inicio de la pandemia.

El control de la replicación viral y la mejoría inmunológica que se logra con el tratamiento anti-retroviral (TARV) combinado de alta eficacia (TAE) ha disminuido la morbilidad y mortalidad de la infección. El TARV eficaz suprime la replicación viral hasta niveles indetectables y previene la aparición de resistencias a los fármacos anti-retrovirales (ARV). Como resultado, los niños infectados por transmisión vertical (más de $90 \%$ de las infecciones en pediatría) viven ahora en la tercera y cuarta décadas de vida ${ }^{1-5}$.

Sin embargo, los niños deben recibir fármacos durante toda su vida, con formulaciones no siempre adaptadas a las edades, que generan intolerancias, toxicidades y cansancio, que los expone al rechazo y por lo tanto, a dificultades en la adherencia.

La mala adherencia tiene relación directa con la falla virológica; determina concentraciones plasmáticas de ARV sub terapéuticas facilitando el desarrollo de resistencia a uno o más de los fármacos que venía recibiendo y la posibilidad de resistencia cruzada a otros anti-retrovirales de la misma familia. Múltiples factores como la potencia del régimen anti-retroviral recibido, farmacocinética, interacciones farmacológicas, fitness viral y el tipo de barrera genética de los ARV utilizados influyen en la relación adherencia-resistencia de los niños infectados. Además la resistencia y la mala adherencia limitan la eficacia de futuros planes terapéuticos ${ }^{1-7}$.

Más de 1.000 nuevas infecciones pediátricas por VIH ocurren cada día, principalmente a través de la transmisión materna infantil (TMI), debida sobre todo al acceso tardío o inadecuado a los servicios de la salud. Sin embargo, una
${ }^{1}$ Centro de Referencia ObstétricoPediátrico VIH-SIDA del Centro Hospitalario Pereira Rossell.

Los autores declaran no tener conflictos de intereses reales ni potenciales.

No existieron fuentes de financiamiento.

Recibido: 16 de noviembre de 2017

Aceptado: 31 de julio de 2018

Correspondencia a:

Virginia González

vickygc21@gmail.com 
proporción pequeña de niños se infectan a pesar de recibir profilaxis para la TMI (PTMI). El uso de diferentes ARV, sobre todo, inhibidores no nucleosídicos y nucleosídicos de la transcriptasa inversa (INNRTy INRT) durante el embarazo, parto o lactancia, expone a los niños al riesgo de adquirir mutaciones de resistencia en el caso de infectarse con VIH (resistencia primaria) ${ }^{8-13}$.

Uruguay tiene una prevalencia de personas infectadas de $0,5 \%$; se estima que a diciembre de 2015 existían unas 12.000 personas con infección por VIH en el país. La tasa de infección en mujeres embarazadas en 2014 fue de $0,29 \%$ y la de transmisión vertical fue de $1,6 \%$ en el año $2013,2,9 \%$ en el 2014 y $1,7 \%$ en el $2015^{16,17}$.

En el Centro Hospitalario Pereira Rossell (CHPR) de Montevideo-Uruguay, prestador que pertenece al subsector público del sistema nacional integrado de salud, existe desde 1990 el Centro Nacional de Referencia Obstétrico Pediátrico VIH-SIDA que asiste a la mujer embarazada infectada con VIH y a sus hijos. Este Centro se ocupa de la asistencia de más de $90 \%$ de los niños infectados del país (de prestadores públicos y privados) y tiene como objetivos fundamentales evitar la TMI del VIH y, en caso de transmisión, la atención del niño y adolescente ${ }^{18}$. A diciembre de 2016 se controlaron en el centro 92 niños y adolescentes bajo 15 años de edad en TARV.

En el año 2008 comenzaron a realizarse en Uruguay los primeros test de resistencia por genotipificación, recomendándose desde entonces, a todo niño infectado previo al inicio del tratamiento o a quienes mostraran fallas al TARV ${ }^{19}$.

Con la información obtenida entre los años 2008 y 2011, se observó en todos los pacientes infectados, niños y adultos, la presencia de una o más mutaciones de resistencia en $82,7 \%$ para cualquiera de las familias antivirales (INRT, INNRT e inhibidores de proteasa-IP) ${ }^{20}$.

Hasta el momento no se han publicado en Uruguay estudios epidemiológicos sobre resistencias en pediatría ni en adultos.

La Organización Mundial de la Salud (OMS) está preocupada por la aparición de VIH resistentes a los ARV (VIHR) y ha propuesto una serie de elementos centinelas de riesgo para detectar precozmente y lograr disminuir este problema de Salud Pública ${ }^{21,22}$.

Se calcula que en el año 2010, 7\% de las personas que iniciaron TARV en los países en desarrollo tenían VIH fármaco-resistente. En los países desarrollados, la cifra era de 10 a $20 \%$. Recientemente, algunos países han comunicado tasas de resistencia de $15 \%$ o más en quienes comienzan el tratamiento contra el VIH y de hasta $40 \%$ en quienes lo reinician.

El aumento de la resistencia tiene importantes repercusiones económicas, dado que los fármacos de segunda y tercera línea son más caros que los de primera línea.

Desde septiembre de 2015, la OMS recomienda que todos los pacientes con infección por VIH sean tratados con anti-retrovirales. Se prevé que el aumento del uso de estos fármacos aumente la resistencia a ellos en todas las regiones del mundo. Para mantener la eficacia a largo plazo de los TARV de primera línea es esencial que se siga vigilando la resistencia y se reduzca al mínimo su aparición y propagación ${ }^{23,24}$.

\section{Objetivo general}

Conocer la prevalencia de resistencia a los ARV en niños y adolescentes uruguayos bajo 15 años de edad, infectados con el VIH, que se controlan en el Centro Nacional de Referencia obstétrico- pediátrico VIH-SIDA del Centro Hospitalario Pereira Rossell (CHPR), entre los años 2008 y 2016.

\section{Objetivos específicos}

- Cuantificar mutaciones de resistencia primarias y secundarias en niños y adolescentes.

- Identificar variables asociadas a resistencias.

- Describir si la información obtenida en el test de resistencia contribuyó a lograr una carga viral indetectable.

\section{Metodología}

Se realizó un estudio descriptivo, de seguimiento longitudinal, a partir de la historia clínica de los niños y adolescentes que se controlan en el Centro Nacional de Referencia Obstétrico- Pediátrico VIH-SIDA del CHPR, Montevideo-Uurguay.

Se incluyeron todos los niños y adolescentes infectados con VIH bajo 15 años de edad, que se asistieron en el Centro como usuarios y que contaban con el resultado de test de resistencia realizado en el período $1 / 1 / 2008$ a 15/12/2016.

Las variables registradas fueron maternas y del niño.

- Variables maternas: momento del diagnóstico de infección por VIH (previo al embarazo, durante el embarazo o parto, luego del parto), TARV recibido previo, durante y luego del embarazo; lactancia materna (tiempo), test de resistencia y resultados, adherencia y tolerancia a los ARV constatadas en la historia clínica.

- Variables del niño/adolescente: edad, sexo, fecha de nacimiento, profilaxis para la TMI recibida, vía de transmisión del VIH, edad al diagnóstico de infección, edad a la que se realizó el primer test de resistencia y su resultado, edad de inicio del TARV, plan ARV de inicio, CV y población linfocitaria CD4+ previo al inicio del TARV, cambio de plan ARV luego de resultado del test de resistencia, CV y LT CD4+ a los 6 meses de iniciado el primer plan ARV y del cambio de plan; adherencia y tolerancia a los ARV constatadas en la historia clínica y eventos clínicos tras el cambio de TARV (SIDA y muerte). 
Las indicaciones autorizadas para su solicitud en el período 2008- 2016 fueron:

- Niños infectados, previo al inicio del tratamiento y

- Niños tratados con ARV que presentan fallo virológico, clínico y/o inmunológico.

Para la realización del test es imprescindible una CV $\geq 1.000$ copias $/ \mathrm{ml}$.

El Departamento de Laboratorios de Salud Pública (DLSP) de Uruguay realiza los tests de genotipificación de VIH-1 para la detección de mutaciones de resistencia a fármacos ARV. El método comercial utilizado desde 2008 a 2013 para la prueba de genotipado del VIH-1 fue Trugene HIV-1 Genotyping Assay ${ }^{\circledR}$ (Siemens Health Care Diagnostics, NY, USA) . Se realizó la extracción de ARN, amplificación mediante reacción de polimerasa en cadena-transcriptasa reversa-RPC-TR, luego secuenciación bidireccional CLIPTM en electroforesis de poliacrilamida y finalmente analizado por GeneObjects. Los programas utilizados para el genotipado del VIH-1 fueron: GeneObjects ${ }^{\mathrm{TM}} 4.1$ (2008/05/26) / TRUGENE_HIV1_G17.gnl (2011/07/15). ADN. DR 4.5 (2011/11/16) GuideLines ${ }^{\text {TM }} 17.0$ (2011/06/11)@ 2012 Siemens Healthcare Diagnostics ${ }^{25}$. Desde 2014 a la fecha se realiza la genotipificación del VIH-1 en base al protocolo propuesto por Alemán y cols. ${ }^{26}$, con secuenciación en Macrogen, South Korea y análisis bioinformáticos en el Departamento de Laboratorios de Salud Pública, según la base de datos de la Universidad de Stanford (HIV Drug Resistance Database) $)^{27}$.

\section{Definiciones}

Se consideró resistencia primaria a la encontrada en niños sin uso previo de ARV (naive) con fines terapéuticos, por lo que se incluyeron en este grupo los niños que recibieron zidovudina (AZT) o AZT más nevirapina (NVP) como parte de la profilaxis de la TMI. Se consideró resistencia secundaria a la encontrada en niños quienes habían recibido unos o más planes terapéuticos con ARV. Se definió buena adherencia a la registrada por el médico tratante en la historia clínica (asiduidad de los controles, repetición de recetas de ARV, retiro de ARV en farmacia y el relato de los padres y niños sobre su adherencia).

El estudio fue autorizado por el Comité de Ética del CHPR.

\section{Análisis estadístico}

Para el análisis de los resultados se utilizaron medianas como medida de tendencia central y porcentajes con su respectivo intervalo de confianza del 95\%. Se utilizó el test de $\chi^{2}$ para comparar ambos grupos y el Programa EpiInfo Versión 6.04.

\section{Resultados}

Se incluyeron 56 niños y adolescentes con una mediana de edad al momento del diagnóstico de cuatro meses (rango 27 días-12 años). El 55\% ${ }^{31}$ eran varones y 95\% $(53 / 56)$ se infectó por transmisión vertical.

Se solicitó test de resistencia previo al inicio del tratamiento en 36 niños (64\%) y por fallo terapéutico en 20 (36\%). Los resultados se muestran en la Figura 1.

La mediana de edad a la que se realizó el primer test de resistencia fue de cinco meses (rango 2 meses-12 años) para la búsqueda de resistencias primarias y de nueve años (rango 1-14 años) para resistencias secundarias.

Las madres de los 53 pacientes infectados por transmisión vertical presentaban las siguientes características:

- Ninguna tenía realizado test de resistencia durante el embarazo.

- 15 conocieron su infección durante el embarazo y 10 durante el parto.

- 1 recibía TARV previo al embarazo.

- $14(26 \%)$ lo iniciaron durante el embarazo

- $19(36 \%)$ recibieron ARV durante el parto.

De las mujeres con tratamiento durante el embarazo, se registró mala adherencia en 3 casos $(20 \%)$.

Las variables maternas se muestran en la Tabla 1.

\section{Resultados de resistencias}

La resistencia total identificada fue de $28,6 \%$ (IC 95\% $17,7-42,4)$ correspondiendo a 16 niños que presentaban mutaciones de resistencia: cuatro de 36 niños con mutaciones primarias y 12 de 20 con resistencias secundarias.

La Figura 2 muestra los resultados de los test de resistencia y las Tablas 1 y 2 las mutaciones encontradas en los 16 niños.

\section{Resistencias primarias}

De los 36 niños con test de resistencia previo al tratamiento, se identificaron cuatro niños $(11,1 \%)$ con resistencia primaria: uno con resistencia a los INNRT,

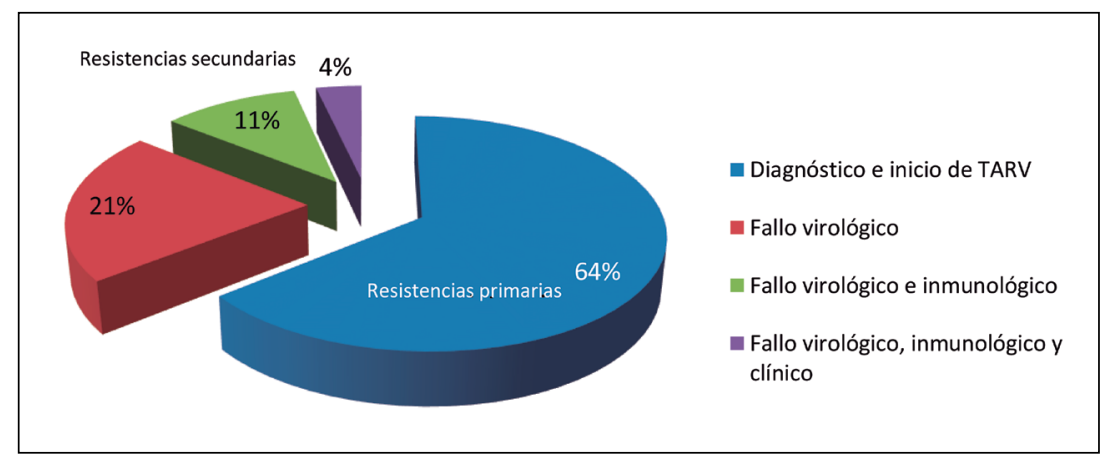

Figura 1. Motivos de solicitud de test de resistencia $(n=56)$. 


\begin{tabular}{|c|c|c|c|c|c|c|c|c|c|}
\hline & \multirow[t]{2}{*}{ Madres } & & \multicolumn{3}{|c|}{$\begin{array}{l}\text { Profilaxis en el niño } \\
\qquad(n=36)\end{array}$} & \multirow[t]{2}{*}{$\begin{array}{l}\text { Mutaciones de } \\
\text { resistencia }\end{array}$} & \multirow[t]{2}{*}{$\begin{array}{l}\text { ARV a los que es } \\
\text { resistente }\end{array}$} & \multirow[t]{2}{*}{$\begin{array}{l}\text { Sin mutaciones } \\
\text { de resistencia }\end{array}$} & \multirow[t]{2}{*}{ Totales } \\
\hline & & & AZT v/o & $\begin{array}{c}\text { AZT + } \\
\text { NVP v/o }\end{array}$ & $\begin{array}{l}\text { Sin } \\
\text { profilaxis }\end{array}$ & & & & \\
\hline \multirow{7}{*}{ 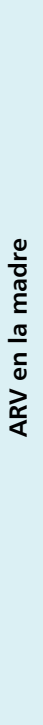 } & \multirow[t]{2}{*}{ Parto } & $\begin{array}{l}\text { AZT } i / v+1 \text { dosis } \\
\text { NVP } v / o\end{array}$ & 1 & & & $\begin{array}{c}\text { RT: E138K, PR: } \\
\text { M36I, K43T. }\end{array}$ & $\begin{array}{c}\text { Posible RESISTENCIA } \\
\text { A EFV, NVP, ETV } \\
\text { Y TPV }\end{array}$ & & \multirow[t]{2}{*}{9} \\
\hline & & AZT i/v & 6 & 2 & & $\begin{array}{c}\text { * RT: K101Q, } \\
\text { K103N, PR: M36I. }\end{array}$ & $\begin{array}{c}{ }^{*} \text { RESISTENCIA A } \\
\text { NVPY EFV }\end{array}$ & 7 & \\
\hline & $\begin{array}{l}\text { Previo al embarazo } \\
+ \text { Embarazo }\end{array}$ & $\mathrm{AZT}+3 \mathrm{TC}+\mathrm{SQV} / \mathrm{r}$ & 1 & & & $\begin{array}{c}\text { RT: M41L, T69D, } \\
\text { L100I, K103N, } \\
\text { M184V, T215F, PR: } \\
\text { K20R, D30N, L33F, } \\
\text { M36I, N88D, L90M }\end{array}$ & $\begin{array}{c}\text { RESISTENTE A : 3TC, } \\
\text { EFV, NVP, ATV, IND, } \\
\text { NVF, POSIB: ABC, } \\
\text { DDI, D4T, TDF, AZT, } \\
\text { ETR, SAQ. }\end{array}$ & & 1 \\
\hline & \multirow{2}{*}{$\begin{array}{l}\text { Embarazo + parto } \\
(A Z T \mathrm{i} / \mathrm{v})\end{array}$} & $A Z T+3 T C+L o p / r$ & 7 & \multirow[t]{2}{*}{1} & & & & 8 & \multirow[t]{2}{*}{9} \\
\hline & & $\mathrm{AZT}+3 \mathrm{TC}+\mathrm{SQV} / \mathrm{r}$ & 1 & & & & & 1 & \\
\hline & Sin profilaxis & & 3 & 1 & 13 & $\begin{array}{c}\text { * *IP: I15V, K20R, } \\
\text { M36I, D60E, I62V, } \\
\text { T74S, L89M. }\end{array}$ & $\begin{array}{c}{ }^{* *} \text { Resistente a } \\
\text { SQV/r }\end{array}$ & 16 & 17 \\
\hline & Totales & & 19 & 4 & 13 & & 4 & 32 & 36 \\
\hline
\end{tabular}

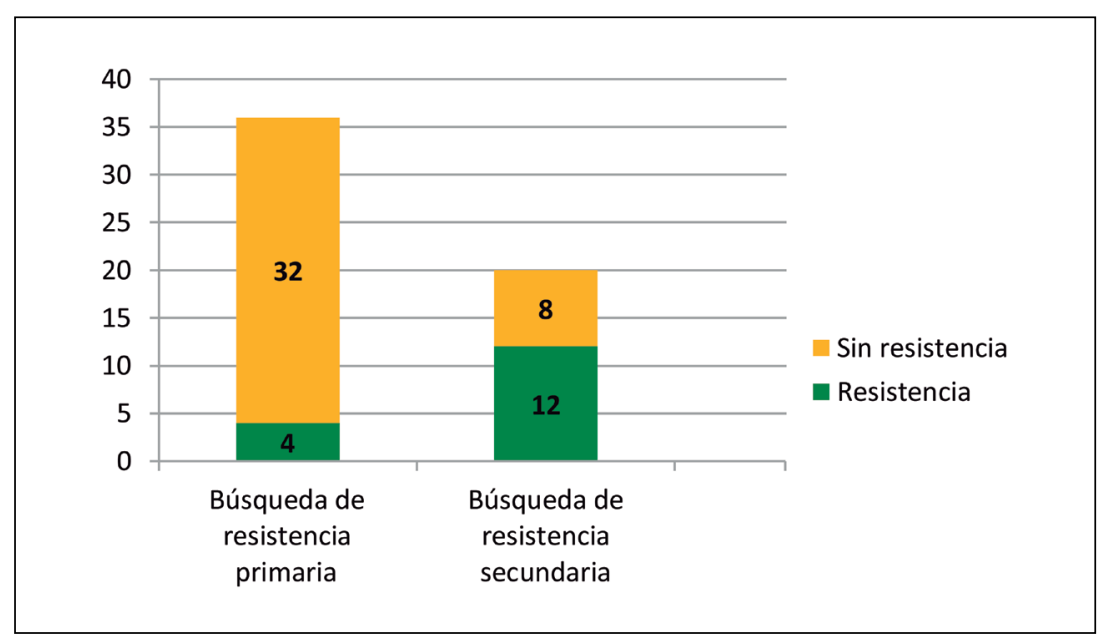

Figura 2. Resultados de los tests de resistencia solicitados $(n=56)$.

uno con resistencia a INNRT y a tipranavir, uno a INNRT, INRT e IP y uno con resistencia a SQV/r. La exposición a ARV maternos, la profilaxis recibida por los 36 niños y las mutaciones de resistencia se muestran en la Tabla 1.

\section{Resistencias secundarias}

De los 20 niños con fallo terapéutico, ocho presentaban adherencia adecuada y cuatro de ellos tuvieron mutaciones de resistencia. De los 12 con mala adherencia, ocho tenían mutaciones de resistencia. La adherencia y presencia de mutaciones de resistencia secundaria se muestran en la Tabla 3.

En este pequeño grupo no se encontró asociación entre la mala adherencia y la presencia de mutaciones de resistencia $(p>0,05)$.

Los ARV más comprometidos fueron: lamivudina (3TC) y emtricitabina (FTC) 83\% (10/12); efavirenz (EFV) y NVP en 58\% (7/12).

Uno de los niños presentó resistencia los INNTR a pesar de no haber sido expuesto a esa familia de ARV. El resto presentó resistencias a las familias expuestas durante los diferentes planes terapéuticos. Los ARV recibidos por los 12 niños con presencia de resistencias y las mutaciones encontradas se presentan en la Tabla 2.

\section{Resultados del tratamiento empírico inicial y luego del test de resistencia}

El plan terapéutico inicial fue AZT/ 3TC y Lop/r en $52 \%(29 / 56)$ de los casos y 46\% (26/56) presentó mala adherencia a este plan. 


\begin{tabular}{|c|c|c|c|c|c|c|}
\hline \multirow{2}{*}{$\begin{array}{l}\text { Edad (años) } \\
\text { al test de } \\
\text { resistencia }\end{array}$} & \multicolumn{4}{|c|}{ Plan ARV recibido previo al test de resistencia } & \multirow[t]{2}{*}{ Mutaciones } & \multirow[t]{2}{*}{ ARV a los que es resistente } \\
\hline & Primer plan & Segundo plan & Tercer plan & Cuarto plan & & \\
\hline 8 & $\begin{array}{l}\mathrm{AZT}+3 \mathrm{TC} \\
+\mathrm{Nel}\end{array}$ & $\begin{array}{c}\text { D4T }+ \text { EFV }+ \\
\text { Lop/r }\end{array}$ & & & $\begin{array}{c}\text { RT: K103N, G190A, PR: L10I, 115V, } \\
\text { M36L, I54V, D60E, I62V, L63P, V82A, } \\
\text { L89M }\end{array}$ & $\begin{array}{l}\text { NVP, EFV, NFV, APV/R, FPV/r; Posible } \\
\text { resis: IDV/r, Lop/r, ATV }\end{array}$ \\
\hline 12 & $\begin{array}{l}\mathrm{AZT}+3 \mathrm{TC} \\
+\mathrm{Nel}\end{array}$ & $\begin{array}{l}\mathrm{D} 4 \mathrm{~T}+\mathrm{ABC} \\
\quad+\mathrm{EFV}\end{array}$ & & & $\begin{array}{c}\text { RT: D67N, V90I, K103N, V108I, K219R, } \\
\text { P225H }\end{array}$ & Efavirenz, posible resistencia d4T \\
\hline 7 & $\begin{array}{l}\text { AZT }+3 T C+ \\
\text { Nelf }+ \text { Lop } / r\end{array}$ & & & & $\begin{array}{l}\text { RT: M41L, D67N, T69D, K70R, M184V. } \\
\text { PR: L10F, I13V, K20I, L24I, M36I, K43T, } \\
\text { M46I, I54V, I62V, T74S, V82A }\end{array}$ & $\begin{array}{c}\text { 3TC y ABC, SQV/r, IDV, NFV, APV, } \\
\text { FPV, Lop/rit, ATV. Posible resistencia } \\
\text { AZT,DDI, d4T y TDF, TPV }\end{array}$ \\
\hline 13 & $\begin{array}{l}A Z T+3 T C+ \\
\quad \text { Nelf }\end{array}$ & $\begin{array}{c}\text { AZT }+3 \mathrm{TC}+ \\
\mathrm{Lop} / \mathrm{r}\end{array}$ & & & RT: M184V, PR: M36I & 3TC/FTC \\
\hline 12 & $\begin{array}{l}\text { AZT }+3 T C+ \\
\text { Nelf }+ \text { Lop } / r\end{array}$ & $\begin{array}{c}\text { AZT }+3 \mathrm{TC}+ \\
\mathrm{Lop} / \mathrm{r}\end{array}$ & $\begin{array}{l}\mathrm{AZT}+3 \mathrm{TTC} \\
\quad+\mathrm{EFV}\end{array}$ & & RT: K103N, V108I, M184V & Efavirenz, nevirapina y 3TC \\
\hline 9 & $\begin{array}{l}A Z T+3 T C+ \\
\quad \text { Nelf }\end{array}$ & $\begin{array}{l}\text { AZT }+3 \mathrm{TC}+ \\
\mathrm{Lop} / \mathrm{r}\end{array}$ & $\begin{array}{l}\mathrm{AZT}+3 \mathrm{TC} \\
\quad+\mathrm{EFV}\end{array}$ & & $\begin{array}{c}\text { RT: D67N, K70R, V75M, K103N, } \\
\text { M184V, G190A, K219Q. PR: L10V, } \\
\text { M36l. }\end{array}$ & 3TC/FTC, AZT, D4T, EFV, NVP \\
\hline 9 & $\begin{array}{l}A Z T+3 T C+ \\
\quad L o p / r\end{array}$ & & & & RT: M184V, PR: M36I & 3TC/FTC \\
\hline 1 & $\begin{array}{c}A Z T+3 T C+ \\
\quad L o p / r\end{array}$ & & & & TR: M184V & 3TC/FTC \\
\hline 4 & $\begin{array}{c}\text { AZT }+3 T C+ \\
\quad \text { Lop } / r\end{array}$ & & & & TR: M184V & 3TC/FTC \\
\hline 14 & $A Z T+3 T C$ & $\begin{array}{l}\text { 3TC }+ \text { DDC }+ \\
\text { Nelf }\end{array}$ & $\begin{array}{l}A B C+N V P \\
\quad+\text { Rit }\end{array}$ & $\begin{array}{l}\mathrm{D} 4 \mathrm{~T}+\mathrm{ABC} \\
\quad+\mathrm{Lop} / \mathrm{r}\end{array}$ & $\begin{array}{c}\text { RT: K103N, V108I, M184V, T215Y, } \\
\text { M23OL, PR: L10I, K20I, M36I, A71T, } \\
\text { T74S, V82A. }\end{array}$ & $\begin{array}{c}\text { 3TC, EFV, NVP, IDV, NFV, SQV/R, POSIB: } \\
\text { ABC, D4T, AZT, ETR, APV/FOSAM/R, } \\
\text { ATV, IDV/R, LOP/R }\end{array}$ \\
\hline 11 & $\begin{array}{l}\text { AZT }+3 T C+ \\
\quad L o p / r\end{array}$ & $\begin{array}{l}\mathrm{AZT}+3 \mathrm{TC}+ \\
\quad \mathrm{EFV}\end{array}$ & & & $\begin{array}{c}\text { RT: L10I, K103N, M184V, PR: K20R, } \\
\text { M36I. }\end{array}$ & 3TC, EFV, NVP, posible resiste a ETV \\
\hline 3 & $\begin{array}{c}A Z T+3 T C+ \\
\quad \text { Nelf }\end{array}$ & $\begin{array}{c}\text { AZT }+3 \mathrm{TC}+ \\
\mathrm{Lop} / \mathrm{r}\end{array}$ & & & Multirresistencia & $\begin{array}{l}\text { A los INRT, INNRT e IP, posible resis- } \\
\text { tencia EFV, sensible solo a DRV/R }\end{array}$ \\
\hline $\begin{array}{l}\text { ABC: Abacavir } \\
\text { FPV/r: FosampI } \\
\text { TDF: Tenofovir: }\end{array}$ & $\begin{array}{l}\text { PV: Amprena } \\
\text { ivir/ritonavir; } \\
\text { V: Tipranavir. }\end{array}$ & $\begin{array}{l}\text { ATV: Atazan } \\
\text { : Emtricitavina } \\
\text { s otros } 8 \text { niño }\end{array}$ & $\begin{array}{l}\text { AZT: Zido } \\
\text { : Indinavir }\end{array}$ & $\begin{array}{l}\text { DDI: D } \\
\text { amivudin }\end{array}$ & $\begin{array}{l}\text { na; D4T: Stavudina; DRV/r: Darunav } \\
\text { : Lopinavir/ritonavir; NVF: Nelfinavir; }\end{array}$ & $\begin{array}{l}\text { navir; EFV: Efavirenz; ETV: Etravirina } \\
\text { Nevirapina; SQV/r: Saquinavir/ritonavir }\end{array}$ \\
\hline
\end{tabular}

Tabla 3. Resistencia secundaria y adherencia en niños con falla terapéutica

\begin{tabular}{|c|c|c|c|c|}
\hline & & \multicolumn{2}{|c|}{$\begin{array}{l}\text { Presencia de resistencia } \\
\text { secundaria }\end{array}$} & \multirow[t]{2}{*}{ Total } \\
\hline \multirow{3}{*}{$\begin{array}{l}\text { Adherencia } \\
\text { adecuada }\end{array}$} & & Sí & No & \\
\hline & Sí & 4 & 4 & 8 \\
\hline & No & 8 & 4 & 12 \\
\hline $0,56, p>$ & & & & \\
\hline
\end{tabular}

De los 36 niños con búsqueda de resistencias primarias, 12 (33\%) lograron CV indetectable a los seis meses de iniciar el plan terapéutico empírico.

El resultado del test de resistencia cambió el plan ARV en $15(26,7 \%)$ de los 56 niños, uno tenía resistencia primaria, 12 resistencias secundarias y dos sin mutaciones de resistencia pero en fallo terapéutico. El cambio logró obtener CV indetectable a los seis meses en ocho de los 15 niños: siete con resistencias secundarias y uno con resistencias primarias. Los resultados se muestran en la Figura 3. 


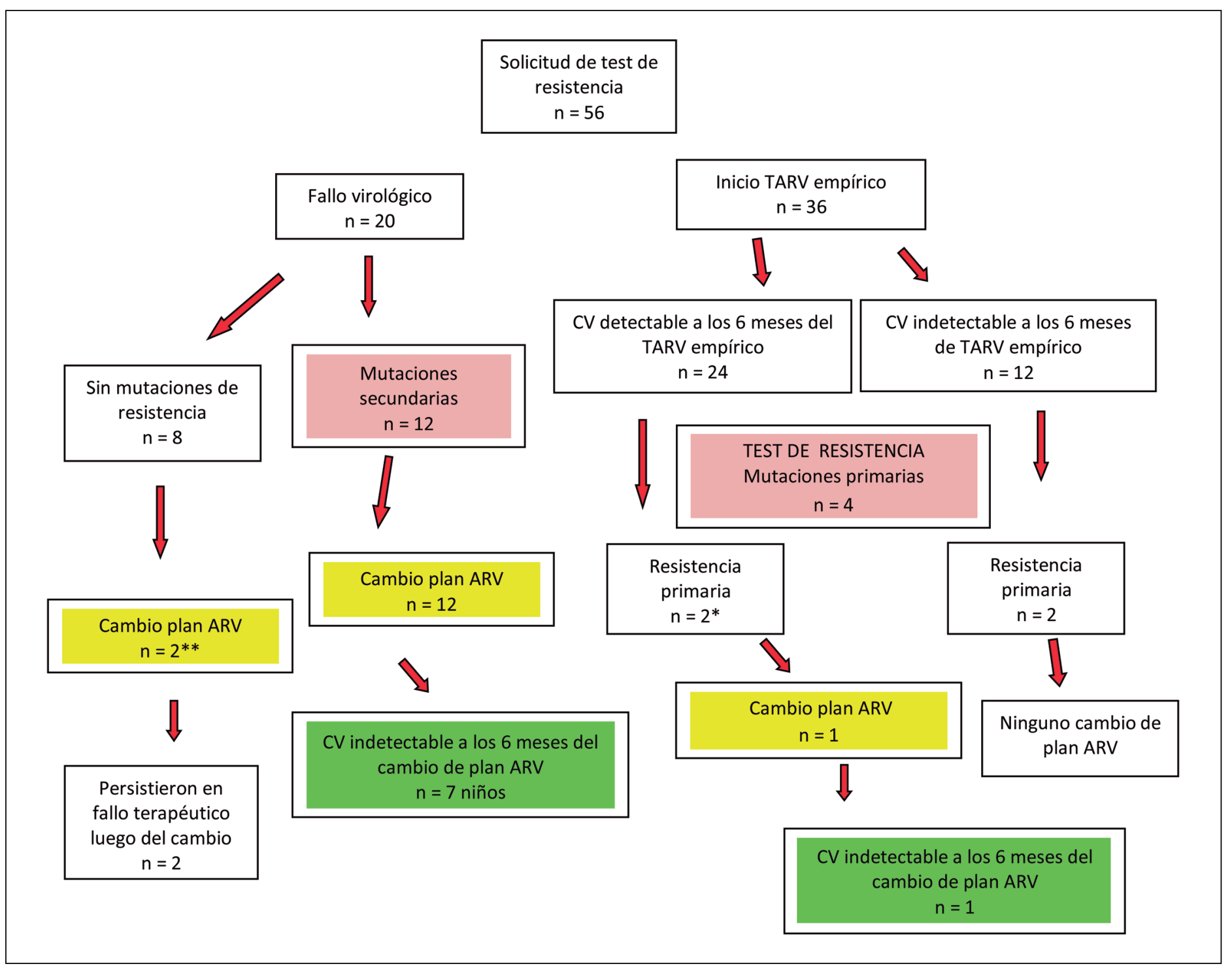

Figura 3. Diagrama de evolución de la carga viral según resultado del test de resistencia $(n=56)$. ${ }^{*}$ n uno de ellos, la resistencia primaria no incluía ARV del tratamiento empírico. **Se indicó cambio para simplificación del TARV.

El cambio de TARV luego del resultado del test de resistencia no se asoció con SIDA o muerte en caso alguno.

Fallecieron dos niños en el período analizado: un varón de 2 años y una niña al año de edad. Ambos eran hijos de madres que no controlaron su embarazo. Los niños iniciaron TARV, pero la adherencia era mala. Ninguno presentaba mutaciones de resistencia primaria.

\section{Discusión}

La resistencia del VIH a los ARV representa uno de los mayores problemas para el éxito del tratamiento de los pacientes infectados. Las consecuencias incluyen el fracaso del tratamiento y la propagación del VIH resistente a fármacos ${ }^{28-30}$.

Este es el primer estudio de prevalecía de resistencias en Uruguay. En nuestro país, 98\% de los niños y adolescentes bajo 15 años de edad, infectados con VIH reciben TARV ${ }^{17}$.

La prevalencia total de mutaciones de resistencia $(28,6 \%)$ y la de resistencias primarias $(11,1 \%)$ en la población analizada es similar a la descrita en la región y en el mundo, pero fue más elevada en los casos de resistencia secundaria $(60 \%)^{23,31-37}$.

La mutación asociada a resistencias primarias más 
frecuente fue la K103N asociada a la resistencia a INNTR y coincidiendo con la mayor exposición a estos ARV en la profilaxis para la TMI.

La mayoría de las mutaciones en las resistencias secundarias fueron: M184V (83\%) y K103N (58\%) debido al uso de INTR (3TC) e INNTR (EFV) en los planes terapéuticos de inicio en Uruguay.

Lu y cols., encontraron en 229 niños chinos en tratamiento ARV, una mayor proporción de mutaciones de resistencia a INTR (M184V/L y M41L) y la asociación de resistencias a INTR y a INNTR en pacientes con fallo terapéutico ${ }^{32}$.

Argentina muestra también las mutaciones M184V y K103N como las más frecuentes en gestantes infectadas con TARV y la K103N en las que no recibieron TARV ${ }^{37}$.

La aparición de resistencia es mayor cuando se busca a niños en fallo terapéutico que cuando se busca antes del inicio del tratamiento empírico. Si bien este estudio no pudo demostrar la asociación entre mala adherencia y resistencia a los $\mathrm{ARV}$, todos los niños que lograron $\mathrm{CV}$ indetectable con tratamiento empírico tenían buena adherencia. En un estudio realizado en el CHPR en el año 2016 sobre la respuesta virológica al TARV en niños bajo dos años de edad, cuyo objetivo era describir el tiempo necesario que requieren estos niños para lograr $\mathrm{CV}$ indetectable luego del inicio del tratamiento ARV y los factores vinculados al fallo virológico, se encontró que de 27 niños incluidos, cinco lograron CV indetectable a los seis meses del inicio empírico del TARV y 11 nunca lograron carga viral indetectable durante el período del estudio. La mala adherencia, toxicidad e intolerancia a las presentaciones pediátricas existentes en el país fueron los motivos que se identificaron con mayor frecuencia en los niños con fallo virológico ${ }^{38}$.

La prevalencia y los patrones de las mutaciones asociadas a la fármaco-resistencia en los pacientes que no responden al TARV de primera línea nos permiten diferenciar entre aquellos que no responden al TARV y no presentan mutaciones de resistencia y que, por lo tanto, se beneficiarían de las medidas para mejorar la adherencia, de aquellos que presentan mutaciones asociadas a resistencia. En estos últimos se debe valorar diferentes escenarios que pueden haber contribuido al desarrollo de resistencia: el tiempo que ha estado sometido a ese régimen de primera línea; los regímenes de tratamiento empleados y su tolerancia; y los subtipos del VIH. La valoración individual de los resultados nos permitirá ajustar el tratamiento ideal para cada niño con los recursos disponibles ${ }^{35}$.

En este estudio, el cambio de plan terapéutico se realizó a los 12 niños que mostraron resistencias secundarias y a dos que no mostraron resistencias pero necesitaron simplificar el TARV para mejorar la adherencia. A pesar de estas consideraciones, siete niños persistieron en falla terapéutica a los seis meses del cambio de plan, lo que muestra que debemos seguir trabajando en la adherencia de los niños y para eso debemos simplificar y buscar presentaciones que sean adecuadas a cada niño y a cada familia.

La prevalencia de resistencia primaria en este estudio fue de $11,1 \%$.

En diversos trabajos se ha evaluado la prevalencia de mutaciones de resistencia en niños que reciben diferentes regímenes de prevención de la TMI del VIH. En el año $2010,48 \%$ de las mujeres embarazadas infectadas por el VIH en los países de ingresos bajos y medianos recibieron tratamiento preventivo de la TMI, del cual estaba excluida NVP en dosis única.

El uso generalizado de INNTI en las mujeres embarazadas o que amamantan, ya sea para prevenir la TMI o como TARV, puede ocasionar que los niños se infecten con un VIH resistente a los INNTI o bien resistente a los INTI, lo cual comprometerá su posterior respuesta al TARV ${ }^{35}$. El empleo de terapias subóptimas y la toma de ARV de baja barrera genética durante el embarazo, el uso de monoterapia o dosis única durante el parto y la profilaxis al recién nacido con mono o biterapia, exponen a los lactantes infectados al desarrollo de mutaciones de resistencia primaria.

De los cuatro niños con resistencia primaria, dos eran hijos de madres que se expusieron a alguno de los ARV que mostró resistencia, una durante el embarazo con mala adherencia y otra durante el parto. Otro niño recibió tres dosis de NVP al nacer. Si bien el número de casos es muy pequeño, la exposición previa en la madre o el RN sobre todo a INNTR podría explicar la presencia de resistencias primarias. Las resistencias a IP en uno de los niños no se vinculó a exposición materna a los ARV por lo que la infección materna durante el embarazo pudo adquirir un virus resistente y transmitir esa resistencia a su hijo infectado.

Yeganeh N y cols., describen la presencia de resistencia a los ARV en mujeres embarazadas no tratadas con ARV y la transmisión de mutaciones de resistencia a sus hijos infectados ${ }^{31}$.

En Brasil, Andrade y cols., encontraron una prevalencia de resistencia primaria de $16,2 \%: 21,1 \%$ en niños expuestos a profilaxis para la TMI y $14,3 \%$ entre los niños no expuestos ${ }^{36}$.

Argentina mostró 56,6\% de mujeres embarazadas naive de TARV con resistencia a los ARV ${ }^{37}$.

Estos hallazgos refuerzan la importancia de la solicitud de test de resistencia previo al inicio de TARV en todos los niños, sin importar la exposión del binomio a los ARV ${ }^{31}$.

El diagnóstico precoz de la infección materna, la adecuada adherencia a los ARV y la CV indetectable son las estrategias adecuadas para evitar la infección del $\mathrm{RN}$ y también para evitar la aparición y transmisión de 
resistencias. Lograr que la madre llegue al parto con $\mathrm{CV}$ indetectable evitaría también la exposición a INNTR previo al nacimiento y en el RN expuesto ${ }^{7,14-15}$.

La TARV empírica de inicio en los niños $<2$ años en Uruguay es AZT/3TC/Lop/r, por lo que la resistencia a INNTR no requiere cambio del plan en esa población. En el estudio, de los 36 niños a los que se les buscó resistencia primaria, 23 no lograron tener CV indetectable a los seis meses del inicio o cambio del TARV, lo que amerita revisar la adherencia al tratamiento en los niños más pequeños, valorar la tolerancia a las presentaciones farmacéuticas y plan de inicio empírico más adecuado en este grupo etario donde el mal control de la replicación viral puede dejar secuelas en su crecimiento y desarrollo.

Los resultados de estudios de prevalencia serán útiles para la toma de decisiones nacionales, regionales y mundiales sobre la selección óptima del tratamiento con ARV de primera línea en los niños y para el manejo de los presuntos fracasos terapéuticos, incluido el uso orientado de la carga viral y la intensificación del apoyo a la adherencia al tratamiento (OMS 2016).

\section{Conclusiones}

La prevalencia de mutaciones primarias es similar a la descrita sobre todo en países desarrollados, mientras que es mayor en los casos de resistencia secundaria.

Los ARV más comprometidos son INNTR, 3TC y FTC.

Los resultados de mutaciones de resistencia lograron cambiar el plan ARV en niños con mutaciones de resistencia secundaria, pero no todos pudieron lograr CV indetectable.

Se debe mantener la vigilancia de mutaciones de resistencia en nuestro país, fortalecer la adherencia a los ARV y seleccionar la terapia individualizada más adecuada para lograr la supresión viral y evitar la transmisión de resistencia.

Agradecimientos. A los profesionales del Departamento de Laboratorios de Salud Pública del Ministerio de Salud. Uruguay, Lic. Maria Brasesco, Dres. Héctor Chiparelli, Rosa Flieller y Dora Ruchansky.

\section{Resumen}

Introducción: A nivel mundial, la tasa global de resistencia primaria y secundaria a los anti-retrovirales (ARV) es de 15 y 40\%, respectivamente. Se desconoce su prevalencia en Uruguay. Objetivo: Conocer la prevalencia de resistencia a los ARV en niños y adolescentes uruguayos bajo 15 años de edad infectados con VIH que se controlan en el Centro Hospitalario Pereira Rossell entre 2008 y 2016. Objetivos especificos: Cuantificar mutaciones de resistencia primarias y secundarias e identificar variables asociadas a resistencias; describir si el resultado del test de resistencia contribuyó a lograr una carga viral (CV) indetectable. Metodología: Descriptivo observacional, seguimiento longitudinal. Se incluyeron menores de 15 años con test de resistencia entre 1 de enero de 2008 y 15 de diciembre de 2016. Variables maternas y del niño. Resultados: Se incluyeron 56 niños. Tenían test de resistencia previo al inicio TARV 36 niños $(64 \%)$ y por fallo terapéutico 20 (36\%). La resistencia total fue $28,6 \%$ (16 niños): cuatro $(11,1 \%)$ con mutaciones primarias y 12 $(60 \%)$ secundarias. El test modificó el plan ARV en 15 $(26,7 \%)$ de los 56 niños. El cambio logró CV indetectable a los seis meses en ocho casos. El cambio de TARV no se asoció con sida o muerte. Discusión: Los estudios de prevalencia son útiles para la toma de decisiones sobre la selección inicial de ARV. La prevalencia de mutaciones primarias fue similar a la publicada, mientras que la secundaria fue mayor.

\section{Referencias bibliográfícas}

1.- Flynn P, Rudy B, Douglas S, Lathey J, Martinez J, Silio M, et al. Virologic and immunologic outcomes after 24 weeks in HIV type 1 -infected adolescents receiving highly active antiretroviral therapy. J Infect Dis 2004; 190 (2): 271-9. https://doi.org/10.1086/421521.

2.- Van Dyke R, Lee S, Johnson G, Wiznia A, Mohan K, Stanley K, et al. Reported adherence as a determinant of response to highly active antiretroviral therapy in children who have human immunodeficiency virus infection. Pediatrics. 2002; 109 (4): e61. http://pediatrics. aappublications.org/content/pediatrics/109/4/ e61.full.pdf?ck=nck.

3.- Watson D, Farley J. Efficacy of and adherence to highly active antiretroviral therapy in children infected with human immunodeficiency virus type 1 . Pediatr Infect Dis J 1999; 18 (8): 682-9. http://dx.doi. org/10.1097/00006454-199908000-00006.

4.- Chadwick E, Rodman J, Britto P, Powell C, Palumbo P, Luzuriaga K, et al. Ritonavir-based highly active antiretroviral therapy in human immunodeficiency virus type 1-infected infants younger than 24 months of age. Pediatr Infect Dis J 2005; 24 (9): 793-800.

5.- Vreeman R, Nyandiko W, Liu H, Tu W, Scanlon M, Slaven J, et al. Measuring adherence to antiretroviral therapy in children and adolescents in western Kenya. J Int AIDS Soc. 2014; 17:19227. http://dx.doi.org/10.7448/ IAS.17.1.19227.

6.- Gardner E, Burman W, Steiner J, Anderson P, Bangsberg D. Antiretroviral medication adherence and the development of classspecific antiretroviral resistance. AIDS 2009; 23 (9): 1035-46. doi: 10.1097/ QAD.0b013e32832ba8ec.

7.- Panel on Antiretroviral Therapy and Medical Management of HIV Infected Children. Guidelines for the use of Antiretroviral Agents in Pediatric HIV Infection. Disponible en: http://aidsinfo.nih.gov/guidelines on 4/20/2016. 
8.- Kijak G, Avila M, Salomón H. Mother-to-child transmission of drug-resistant HIV. Drug Resist Update 2001; 4: 29-37. https://doi.org/10.1054/ drup.2001.0182

9.- Colgrove R, Pitt J, Chung P, Welles S, Japour A. Selective vertical transmission of HIV-1 antiretroviral resistance mutations. AIDS 1998; 12 (17): 2281- 8. https://www.ncbi.nlm.nih.gov/ pubmed/9863870.

10.- Bauer G, Colgrove R, Larussa P, Pitt J, Welles $\mathrm{S}$. Antiretroviral resistance in viral isolates from HIV-1-transmitting mothers and their infants. AIDS 2006; 20(13): 1707-12. https:// www.ncbi.nlm.nih.gov/pubmed/16931934

11.- De Jose M, Ramos J, Álvarez S, Jiménez J, Muñoz-Fernández M. Vertical transmission of HIV-1 variants resistant to reverse transcriptase and protease inhibitors. Arch Intern Med 2001; 161 (22): 2738-9. https://jamanetwork. com/journals/jamainternalmedicine/articleabstract/752251.

12.- Zeh C, Weidle P, Nafisa L, Lwamba H, Okonji J, Anyango E, et al. HIV-1 drug resistance emergence among breastfeeding infants born to HIV-infected mothers during a single-arm trial of triple-antiretroviral prophylaxis for prevention of mother-to-child transmission: a secondary analysis. PLoS Med 2011; 8 (3): e1000430. https://doi.org/10.1371/journal. pmed.1000430.

13.- World Health Organization. Generic Protocol for surveillance of initial drug-resistant HIV-1 among children $<18$ months of age newly diagnosed with HIV. WHO/HIV/2012.17. Disponible en: www.who.int.

14.- Global HIV/AIDS Epidemic Update 2016. Disponible en: http://www.unaids.org/sites/ default/files/media_asset/global/-AIDSupdates-2016.en pdf (Consultado el 12 de octubre de 2016).

15.- Organización Panamericana de la Salud. Eliminación de la transmisión materno infantil del VIH y la Sífilis en las Américas: actualización de 2014. Washington, DC: OPS, 2014.

16.- AIDS/HIV no Mercosul. Boletín Epidemiológico -Revista Mercosur VIH 2015. Uruguay p 1- 4. Disponible en: www.msp. gub.uy/programa/monitoreo-y-evaluación-0 (Consultado el 10 de octubre de 2016).

17.- Dirección General de la Salud. División Epidemiología. Ministerio de Salud. Boletín Día VIH 2016. Uruguay. Disponible en: www.msp.gub.uy/publicaciones/boletínepidemiológico-día-mundial-vihsida. (Consultado el 12 de diciembre de 2016).

18.- Quian J, Gutiérrez S, González V, Visconti A, Sicco G, Murialdo C, et al. Prevención de la transmisión materno-infantil del VIH de tipo 1 en Uruguay 1987-2009. Análisis de las diferentes medidas adoptadas. Rev Chilena Infect 2012; 29 (1): 87-94. http://dx.doi. org/10.4067/S0716-10182012000100014.

19.- Dirección General de la Salud. División Epidemiología. Ministerio de Salud. Infección por Virus de la Inmunodeficiencia Humana (VIH). Pautas para diagnóstico, monitorización y tratamiento antirretroviral. Actualización 2014. Disponible en: http://www.msp.gub.uy/ programa/guías-nacionales.

20.- Dirección General de la Salud. División Epidemiología. Ministerio de Salud. Informe de la situación nacional de VIH/SIDA. Uruguay. Diciembre 2011. Disponible en: http://www. msp.gub.uy/sites/default/files/archivos adjuntos/informe_epidem_VIH_2011_dic.pdf. (Consultado el 30 de mayo de 2016).

21.- Organización Panamericana de la Salud. How will the 3 by 5 initiative deal with HIV drugs resistance? Ginebra, OPS, 2003 (WHO/ HIV/2003.10; http://www.who.int/3by5/ publications/briefs/en/drug_resistance.pdf).

22.- Organización Panamericana de la Salud. Consolidated guidelines on HIV prevention, diagnosis, treatment and care for key Populations key populations. 2016 update. Disponible en: http://www2.paho.org/hq/index. php?option $=$ com_content\&view $=$ article $\&$ id $=95$ 4\&Itemid $=414$ 42\&lang=es.

23.- Petroni A. Revisión. Resistencia primaria de HIV-1: estado de situación en Argentina. Actualizaciones en Sida. Bs As. 2010; 18 (70): 117-34. https://www.huesped.org.ar/wpcontent/uploads/2014/09/ASEI-70-117-134.pdf

24.- Resistencia a los antimicrobianos. Nota descriptiva. Septiembre de 2016. http://www. who.int/mediacentre/factsheets/fs194/es/.

25.- Stanford University. HIV drug resistance database. https://hivdb.stanford.edu/hivdb/bymutations.

26.- Alemán, Y, Vinken L, Kourí V, Pérez L, Álvarez A, Abrahantes Y, et al. Performance of an in-house human immunodeficiency virus type 1 genotyping system for assessment of drug resistance in Cuba. PLoS ONE 2015; 10.2 : e0117176. doi: 10.1371/journal.pone.0117176.

27.- Rhee S Y, Gonzales M J, Kantor R, Betts B J, Ravela J, Shafer R W. Human immunodeficiency virus reverse transcriptase and protease sequence database. Nucleic Acids Res 2003; 31: 298-303. https://www.ncbi.nlm. nih.gov/pmc/articles/PMC165547/

28.- Brenner B, Routy J, Petrella M, Moisi D, Olivera M, Detorio M, et al. Persistence and fitness of multidrug-resistant HIV type-1 acquired in primary infection. J. Virol 2002; 76(4): 1753-61. DOI: 10.1128/JVI.76.4.17531761.2002
29.- Little S, Holte S, Routy J P, Daar E, Markowitz M, Collier A, et al. Antiretroviral-drug resistance among patients recently infected with HIV. N Engl J Med 2002; 347 (6): 385-94. DOI: 10.1056/NEJMoa013552.

30.- Organización Panamerica de la Salud. HIV drug resistance. Disponible en: http://www. who.int/hiv/topics/drugresistance/en/.

31.- Yeganeh N, Kerin T, Ank B, Watts D H, Camarca M, Joao E C, et al. HIV antiretroviral resistence and transmission in mother-infant pairs enrolled in a large perinatal study. Clin Infect Dis 2018; 66 (11): 1770-7. doi: 10.1093/ cid/cix 1104

32.- Lu X, Zhao H, Zhang Y, Wang W, Zhao C, Li Y, et al. HIV-1 drug-resistant mutations and related risk factors among HIV-1 positive individuals experiencing treatment failure in Hebei Province, China. AIDS Res Ther 2017; 14 (1): 4-13. doi: 10.1186/s12981017-0133-3

33.- Kityo C, Sigaloff K C, Boender T S, Kaudha E, Kayiwa J, Musiime V, et al. HIV drug resistance among children initiating first-line antiretroviral treatment in Uganda. AIDS Res Hum Retroviruses 2016; 32 (7): 628-35. doi: 10.1089/AID.2015.0215.

34.- Silverman R A, Beck I A, Kiptinness C, Levine M, Milne R, McGrath C J, et al. Prevalence of pre-antiretroviral-treatment drug resistance by gender, age, and other factors in HIV-infected individuals initiating therapy in Kenya, 2013-2014. J Infect Dis 2017; 216 (12): 1569-78. doi: 10.1093/infdis/jix544.

35.- Organización Panamerica de la Salud. Estrategia Mundial de la Organización Mundial de la Salud para la Vigilancia y el monitoreo de la farmacorresistencia del VIH, 2012. Washington, DC : OPS, 2016. Disponible en: http://www.who.int/hiv.

36.- Andrade S D, Sabidó M, Monteiro W M, Benzaken A S, Tanuri A. Drug resistance in antiretroviral-naïve children newly diagnosed with HIV-1 in Manaus, Amazonas.J Antimicrob Chemother 2017; 72 (6): 1774-83. doi: $10.1093 / \mathrm{jac} / \mathrm{dkx} 025$

37.- Zapiola I, Cecchini D, Fernández Giuliano S, et al. Resistencia de HIV-1 a drogas antirretrovirales en gestantes del Área Metropolitana de Buenos Aires. Medicina (Buenos Aires) 2016; 76: 34954. http://www.medicinabuenosaires.com/ PMID/27959842.pdf.

38.- Domínguez L. Respuesta virológica al tratamiento antirretroviral en niños menores de dos años infectados por el virus de la inmunodeficiencia humana. [Monografía de posgrado] Montevideo-Uruguay: Facultad de Medicina. Clínica Pediátrica "C", 2016. 